\title{
Direct laser writing of three-dimensional network structures as templates for disordered photonic materials
}

\author{
Jakub Haberko, ${ }^{1,2}$ Nicolas Muller, ${ }^{1}$ and Frank Scheffold ${ }^{1, *}$ \\ ${ }^{1}$ Physics Department and Fribourg Center for Nanomaterials, University of Fribourg, Chemin du Musée 3, 1700 Fribourg, Switzerland \\ ${ }^{2}$ Faculty of Physics and Applied Computer Science, AGH University of Science and Technology, aleja Mickiewicza 30, 30-059 Krakow, Poland
}

(Received 6 July 2013; published 14 October 2013)

\begin{abstract}
In the present article we substantially expand on our recent study about the fabrication of mesoscale polymeric templates of disordered photonic network materials [Haberko and Scheffold, Opt. Expr. 21, 1057 (2013)]. We present a detailed analysis and discussion of important technical aspects related to the fabrication and characterization of these fascinating materials. Compared to our initial report we were able to reduce the typical structural length scale of the seed pattern from $a=3.3 \mu \mathrm{m}$ to $a=2 \mu \mathrm{m}$, bringing it closer to the technologically relevant fiber-optic communications wavelength range around $\lambda \sim 1.5 \mu \mathrm{m}$. We have employed scanning electron microscopy coupled with focused ion beam cutting to look inside the bulk of the samples of different heights. Moreover, we demonstrate the use of laser scanning confocal microscopy to assess the real space structure of the samples fabricated by direct laser writing. We address in detail questions about scalability, finite size effects, and geometrical distortions. We also study the effect of the lithographic voxel shape, that is, the ellipsoidal shape of the laser pen used in the fabrication process. To this end we employ detailed numerical modeling of the scattering function using a discrete dipole approximation scheme.
\end{abstract}

DOI: 10.1103/PhysRevA.88.043822

PACS number(s): 42.70.Qs, 61.43.-j, 81.16.Nd

\section{INTRODUCTION}

Structured dielectric materials in three dimensions can exhibit photonic properties that allow control of the propagation of light [1-7]. For crystalline structures, a complete or incomplete photonic band gap emerges and the propagation of light is hindered or even completely suppressed over a certain range of wavelengths. Full photonic band gaps open up for dielectric materials with a sufficiently high refractive index contrast [6]. The change in transport properties is accompanied by a reduction in the local density of states, which results in increased lifetimes for embedded light emitters such as fluorescent molecules [8]. Interestingly, it appears that many of these unique properties are not tied exclusively to crystalline structures [9-17]. In a recent numerical study Florescu et al. demonstrated that particular designer disordered materials can display large, complete photonic band gaps in two dimensions [14]. Mapping hyperuniform point patterns with short-range geometric order into tessellations allows the design of interconnected networks that give rise to enhanced photonic properties. A point pattern is hyperuniform whenever infinite wavelength density fluctuations vanish [18]. First experimental data obtained for such disordered two-dimensional (2D) structures in the microwave regime have been reported in Ref. [19]. The numerical study by Florescu $e t$ al. has recently been extended to three dimensions by Liew et al. [20]. It was found that for a refractive index $n>3$ (in air) the optical density of states is significantly reduced at certain wavelengths and a photonic band gap opens up [20].

Although some of these concepts, both for crystalline and disordered materials, can be tested on macroscopic length scales using microwaves [21] or ultrasound [22], most of the fundamental interest and possible technological applications concentrate on the infrared or visible range of the optical

\footnotetext{
*Corresponding author: frank.scheffold@unifr.ch
}

spectrum $[2,7,23,24]$. This means that typical structural length scales of the materials need to be scaled down to the submicron range. Periodic photonic structures can be obtained by self-assembly of micro- and nanosized polymer or silica $\left(\mathrm{SiO}_{2}\right)$ spheres resulting in (pseudo)band gaps that can be tuned easily from visible to infrared wavelengths $[5,25]$. Creating complex three-dimensional disordered structures, such as photonic crystals with integrated waveguides [26] or random materials like the ones suggested by Florescu et al. [14], however, mandates the use of more sophisticated top-down fabrication tools. Classical lithography allows sub$100-\mathrm{nm}$ microfabrication but the method is restricted to 2D. In stereolithography, used in modern three-dimensional (3D) printers, thin layers of a photopolymer are exposed according to a sliced representation of the desired structure. Due to the layer-by-layer approach the axial resolution, however, is limited to several micrometers even for the most advanced realizations of this technique [27]. Submicron-scale periodic patterns, such as photonic crystals, can be produced with multibeam interference lithography [28] and proximity field nanopatterning [29]. Probably the most versatile highresolution 3D lithography, however, is two-photon direct laser writing (DLW) $[23,26,30]$. Here, a polymer photoresist absorbs two infrared photons simulateously and the combined energy leads to solidification of the resist. Since the probability for two-photon absorption scales quadratically with laser intensity, the process is highly selective both laterally and axially, providing submicron resolution. The technique has been extended lately towards subdiffraction limited resolution [31], imitating the concept of stimulated emission depletion (STED) known from super-resolution scanning fluorescence microscopy [32,33].

Recently we reported on successful DLW fabrication of mesoscale polymeric templates of the disordered dielectric materials suggested by Florescu et al. [14]. We were able to fabricate hyperuniform network structures with features on 
submicrometer length scales using direct laser writing into a photoresist $[1,34]$. Although the feasibility of fabricating these interesting network structures has been shown, further progress towards the realization of actual photonic materials mandates a better and more detailed understanding of the many aspects that control the material properties and ultimately their optical response. In the present article we substantially expand on our previous study and present a detailed analysis and discussion of some of the most important questions related to the fabrication and characterization of these interesting materials. We employ scanning electron microscopy coupled with focused ion beam cutting to look inside the bulk of the samples. For the routine nondestructive 3D visualization of the structures we use in-house laser scanning confocal microscopy. Compared to our initial study [1], we have succeeded in reducing all relevant length scales by a factor of $5 / 3$ while keeping the quality of the structures unchanged. Moreover, we examine the role of geometric distortions and finite-size effects. We study the effect of the lithographic voxel shape, that is, the ellipsoidal shape of the laser pen used in the fabrication process, as well as macroscopic distortions that emerge as a result of internal stresses in the polymer network during fabrication and development. To this end we analyze in detail numerical calculations of the diffraction patterns of weakly scattering structures using a discrete dipole approximation.

\section{FABRICATION OF MESOSCALE POLYMERIC TEMPLATES BY DIRECT LASER WRITING}

\section{A. Implementation of the laser writing protocol}

Mesoscale polymer network structures can be fabricated by direct laser writing (DLW) using a two-photon polymerization process. Here we fabricate polymeric network structures using the Photonics Professional direct laser writing platform (Nanoscribe GmbH, Germany) [1]. For the fabrication of hyperuniform network structures, we implement a protocol derived by Florescu and coworkers to map a hyperuniform point pattern into tessellations for a photonic material $[14,20]$. As a starting point we use the centroid positions from a maximally randomly jammed assembly of spheres of diameter $a=2-3.3 \mu \mathrm{m}$ with a volume filling fraction of $\phi \simeq 0.64$ [35]. As shown by Torquato and coworkers [36] such seed structures indeed possess the required hyperuniform properties [37]. Next we perform a 3D Delaunay tessellation of the seed pattern: Tetrahedrons are constructed in such a way that no sphere center is contained in the circumsphere of any tetrahedron in the tessellation. The centers-of-mass of neighboring tetrahedrons are then connected, resulting in a 3D random tetrahedral network, Fig. 1. The network structure connection lines are written into a IPG-780 negative tone photoresist (Nanoscribe, Germany). The laser writing pen has an elliptical shape with a typical dimension for the long axis of $800 \mathrm{~nm}$ and $300 \mathrm{~nm}$ for the short axis. In order to expose the photoresist the sample is moved with a three-axis piezo-stage while the position of the pen remains unchanged (stage-scanning configuration). In principle the (static) positioning accuracy of the piezo scanning stage (and thus the pen) is specified to less than $5 \mathrm{~nm}$. However, as explained below, rapid movement over long distances can still
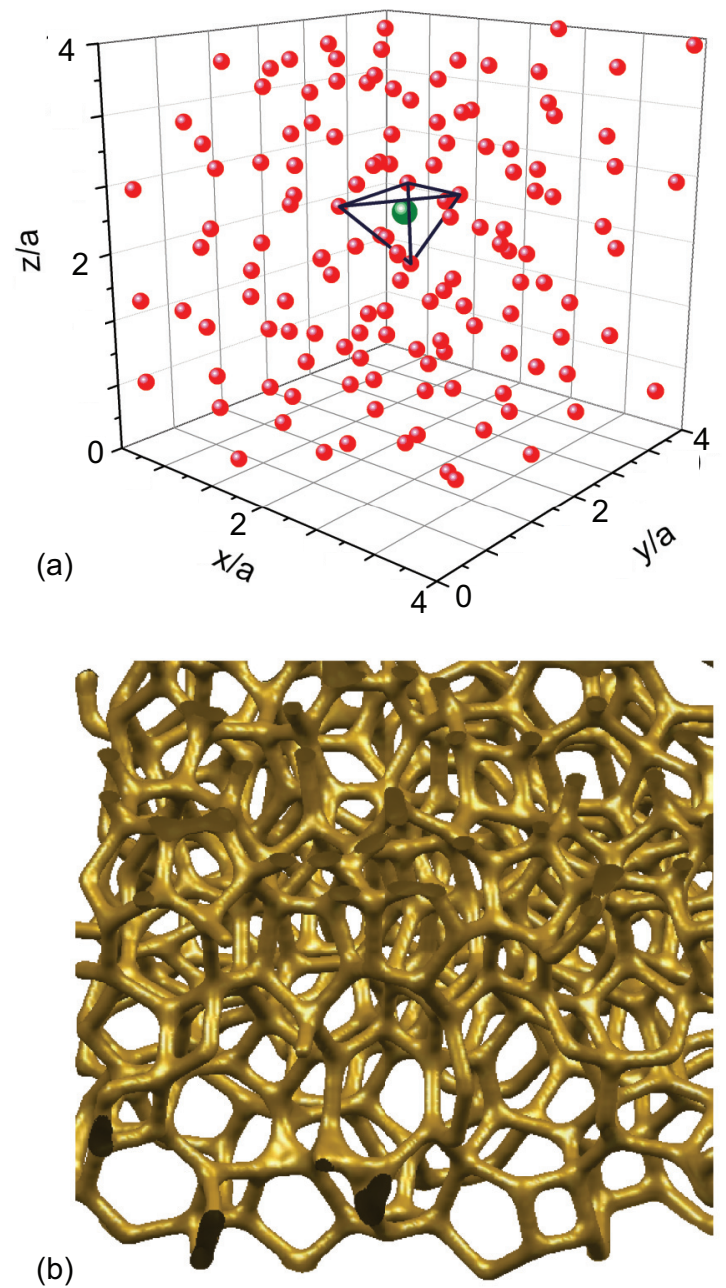

FIG. 1. (Color online) Design of amorphous photonic network structures. (a) The small spheres represent the seed point pattern derived from a jammed assembly of hard spheres with diameter $a$ [35]. The dark lines show an example for the Delauny tesselation of the seed pattern resulting in a network of tetrahedrons. The larger dark sphere shows the centroid position of the tetrahedron. (b) The three-dimensional hyperuniform network obtained by connecting the centroid position of neighboring tetrahedrons and replacing the connection lines with solid rods.

adversely affect the precise positioning of the laser pen in the resist. The overall size of the pen can be adjusted, within a certain range, by adapting the power or residence time of the fs-laser focal spot [39]. Thus the volume-filling fraction of the rods can be controlled by the writing speed or exposure time of the photoresist. In our case the lower acceptable limit is set by mechanical network failure while the upper limit is due to the fact that overexposure will lead to a collapse of the network structure by the fusion of neighboring rods, Fig. 2. The aspect ratio of the pen is only weakly affected by the power setting. Both the approximate size and the aspect ratio are determined by the point spread function of the illuminating microscope objective, which determines the isointensity surface. Above a certain threshold intensity the polymer photoresist is cross linked. The point-spread function in turn is set by the refractive index of about 1.52 of the material and the numerical aperture of the objective, typically 1.4. In the past it was shown that 


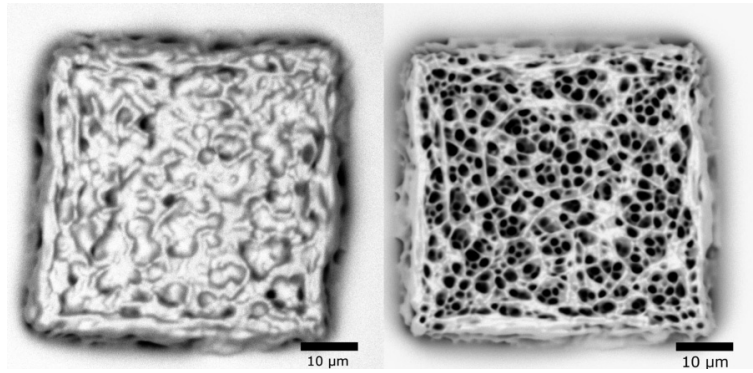

FIG. 2. Electron micrographs of some initial trials to fabricate polymeric network structures by direct laser writing (DLW), illustrating the collapse and deformation of the structures due to an inadequate writing protocol.

placing a shaded ring filter in the beam path can reduce the aspect ratio of the writing pen from $\sim 2.7$ to $\sim 1.8[39,40]$. For fabricating the structures with $a=3.3 \mu \mathrm{m}$ [1], we have used the standard configuration of the DLW system using an oil immersion objective with a numerical aperture 1.4. More recently, for structures featuring a reduction of length scales down to $a=2 \mu \mathrm{m}$, we use dip-in direct laser writing [41,42] using a shaded ring filter [43].

In comparison to the well-established case of periodic rod assemblies [23] the fabrication of these designer disordered structures is very demanding and requires optimization of the writing protocol. To illustrate the complexity of fabricating these structures we show in Fig. 2 a selection of electron micrographs of our failed initial trials. Particular care must be taken how to set up the writing protocol since there are no obvious rules on how to write a random free-standing network structure at optimal resolution. Moreover, it must be ensured that the structure remains mechanically stable in the soft gel photoresist IPG-780 throughout the writing process of about $1 \mathrm{~h}$. As we found out in the course of this work, the difficulties of writing random 3D network structures are mainly due to the hysteresis of the piezoelectric stages and due to problems with unconnected rods. If the stage were to travel rapidly a long distance between two consecutively written rods, this long jump can, presumably due to the inertia of the sample stage, lead to a slightly inaccurate positioning of the second rod. The architecture of the network required that rods must be connected in such a way that in each vertex point four of them meet, Fig. 1. Inaccurate positioning means that some of the rods would have dangling ends rather than being connected to a proper vertex point or worse would not be part of the continuous network altogether. Apart from the deviations of the designed structural properties, this would also adversely affect the network's mechanical stability. Therefore, the writing scheme should favor short distances between consecutively written rods (preferably writing continuous broken lines, whenever possible). Second, if an unconnected rod is written in the bulk of the photoresist, it quickly undergoes severe distortions, Fig. 3. Within seconds after exposure such lines become wiggly and as a result move away from their initial positions. We have been unable to decipher the exact reason for this behavior, but we assume it may result from mechanical stress induced by exposing the photoresist to the writing laser. While this is not a problem for periodically ordered layered structures, it becomes truly challenging when

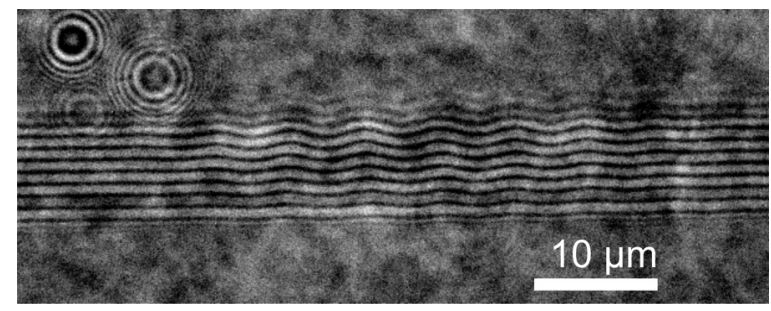

FIG. 3. Light microscopy image taken directly after writing several parallel lines of length $100 \mu \mathrm{m}$ from top to bottom. Writing each line takes $1 \mathrm{~s}$ (at $100 \mu \mathrm{m} / \mathrm{s}$ writing speed) and the wait time between the different lines is $0.2 \mathrm{~s}$. Within seconds after exposure of the photoresist the lines become wiggly and deformed.

fabricating complex disordered networks. Hence, during the writing process each rod should be connected to the substrate or to another rod as quickly as possible.

To overcome these difficulties we divide the writing process into writing subregions. To this end, we select stacks of cubic volumes of side length roughly $1.5 a$ and sort our rods in the following way: (i) once all rods belonging to a certain cube are written, we proceed to a neighboring cube, and (ii) once all cubes closest to the substrate are filled with rods, then the ones lying higher above and so on until the whole network has been written. This procedure solves both the problem of avoiding long piezo movements (strictly) and the issue of connectivity (approximately). An alternative approach might be to identify continuous broken lines connecting all vertices. Among the multitude of possibilities one would then need to choose the one that ensures writing roughly from the substrate upward. However, given the high quality of the samples already obtained by using the first protocol, we have not yet tested the feasibility of the latter approach.

\section{SAMPLE CHARACTERIZATION}

\section{A. Focused ion beam millling and scanning electron microscopy}

Figure 4(a) displays a top-view electron micrograph of a fabricated structure, height $h=8 \mu \mathrm{m}$. The fabrication of this structure has been reported in Ref. [1]. We use ion-beam milling in order to reveal the interior properties of our samples with heights ranging from $h=8$ to $16 \mu \mathrm{m}$. Figures 4(b)-4(d) show snapshots taken during the milling process. The images demonstrate that the structures are written uniformly throughout the sample volume. The images also reveal the size and shape of the ellipsoidal writing pen, which we estimate to $840 \times 280 \mathrm{~nm}^{2}$. The high-resolution bulk imaging allows us to estimate the polymer content in this structure to approximately $10 \%$.

\section{B. Progressive reduction of structural length scales}

For the hyperuniform network structures considered here Liew et al. [20] predict a full band gap to open up at $\lambda \approx 1.77 a$ for $n \simeq 3.6$ and a solid filling fraction of 0.2 . We note that the photoresist used to fabricate the polymer template has a refractive index of only $n=1.52$. If transferred into a high dielectric material such as silicon (with $n \simeq 3.6$ ) the structures reported in our first article [1], derived from a jammed assembly of spheres with a diameter of $a=3.3 \mu \mathrm{m}$, would 

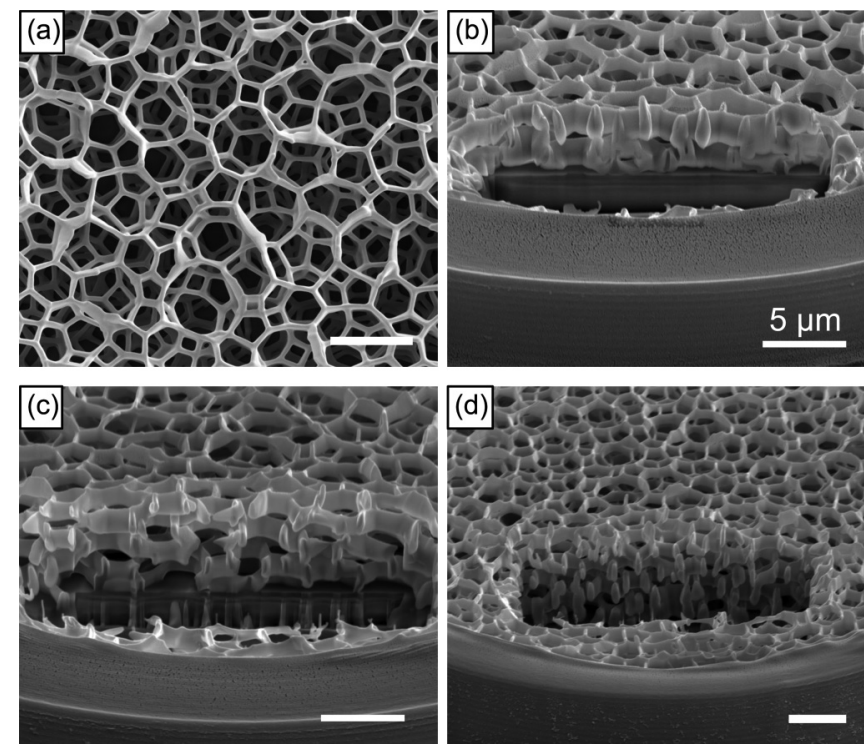

FIG. 4. (a) Scanning electron micrographs of a 3D network structure (height $8 \mu \mathrm{m}$ ), top view. (b) Electron micrograph of the same structure after focused-ion beam milling (oblique view), revealing the internal arrangement of dielectric rods and the glass substrate underneath. (c) Oblique view of a 12- $\mu \mathrm{m}$-high sample. (d) Oblique SEM view of a 16- $\mu$ m-high sample. Scale bars: $5 \mu \mathrm{m}$.

feature a band gap around $\lambda \sim 5.8 \mu \mathrm{m}$. This wavelength is still about four times larger than typical telecommunication wavelengths of $\lambda \sim 1.3-1.65 \mu \mathrm{m}$ [23,24]. In order to shift the bandgap position towards shorter wavelengths, all lengths need to be scaled down. By carefully adjusting the laser writing parameters we succeeded in reducing the structural parameters of our samples by up to a factor of $5 / 3(a=2 \mu \mathrm{m})$ as shown in Fig. 5. In order to routinely assess the structural quality of the polymeric templates we perform optical diffraction experiments with a home-built small-angle light-scattering setup. Experimental details concerning the setup are described in Ref. [1]. The local optical response is characterized by the effective differential cross section $\mathrm{d} \sigma / \mathrm{d} \Omega$ or the scattering function $I(\mathbf{q}) \sim d \sigma / d \Omega$. In the weak scattering limit (or first Born approximation) the scattering function is directly connected to the structural materials properties via a simple Fourier transform $I(\mathbf{q}) \sim\left|\int \rho(\mathbf{x}) e^{-i \mathbf{q} \cdot \mathbf{x}} d \mathbf{x}\right|^{2}$. Here $\rho(\mathbf{x})$ denotes the scattering length density, which in our case is set by the refractive index contrast $\rho(\mathbf{x}) \sim f(\mathbf{x})\left(n / n_{0}-1\right)^{2}$, where $n$ is the polymer refractive index and $n_{0}$ is the refractive index of the surrounding medium [44]. The function $f(\mathbf{x})$ describes the configuration of the polymer network, i.e., $f(\mathbf{x})=1$ in the presence of the polymer and zero otherwise. We find that the scattering pattern, shown in Fig. 6, remains essentially unchanged for the scaled down network structures. This indicates that reducing the length scales by up to a factor $5 / 3$ does not have a negative impact on the optical quality of the structures. As expected the peak value $I\left(q_{\max }\right)$ is observed at $q_{\max } \simeq 2 \pi / a$ under index-matching conditions. For a further reduction of the structural length scales we face some important challenges. The average length $d$ of dielectric rods connecting the nodes of the hyperuniform network is $d=0.39 a$ [20]. Thus for a seed pattern with $a<2 \mu \mathrm{m}$ we
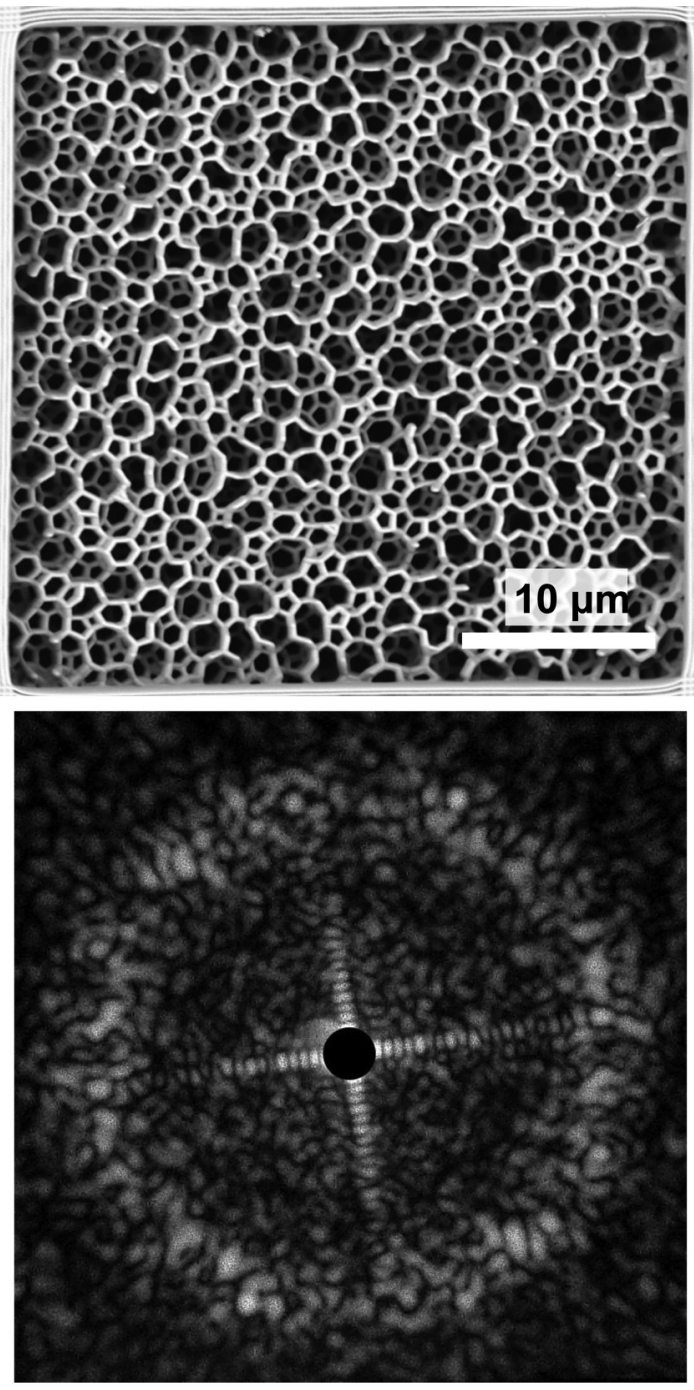

FIG. 5. Upper panel: Scanning electron micrograph of a structure with $a=2 \mu \mathrm{m}$. Lower panel: Experimental light diffraction patterns of the same structure. The lateral size of the image corresponds to a scattering angle of $26 \mathrm{deg}$ (for $\lambda=633 \mathrm{~nm}$ ). The cross-shaped diffraction pattern at lower angles is due to diffraction from the sample boundaries (sample size $40 \times 40 \mu \mathrm{m}^{2}$ ).

would have to write rods $d<0.8 \mu \mathrm{m}$. Moreover, these rods can be oriented arbitrarily in space while the elliptical writing pen has a fixed orientation. Although we still expect to be able to further reduce structural length scales, these estimates show that with the current technology it will be very difficult, if not impossible, to fabricate structures that will feature a bandgap in the range of telecommunication wavelengths. Using STED-inspired DLW [31] would in principle allow a further twofold improved resolution. However, since all of the material and optical aspects of hyperuniform photonic materials can be conveniently studied in the spectral region $\lambda=2-5 \mu \mathrm{m}$, we are currently not exploring such further reduction in length scales until a better understanding of the properties and spectral features has been reached. Moreover other infrared applications in this longer wavelength range, for example, in the field of low-threshold lasing [2,45], could already be targeted. 


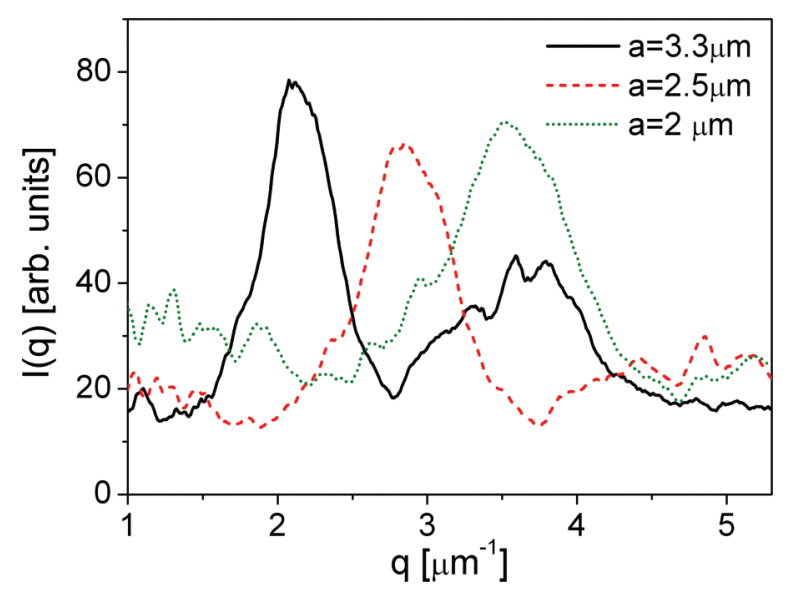

FIG. 6. (Color online) Radially averaged experimental smallangle light-scattering patterns for structures with different scaling parameters $a$.

\section{Three-dimensional optical imaging of hyperuniform polymer network structures}

Focused ion beam milling combined with electron microscopy provides ultra-high-resolution images of the polymer networks. However, the method is destructive, somewhat timeconsuming, and relatively expensive. Diffraction experiments on the other side allow a precise global assessment of the structure quality but are incapable of probing distortions and heterogeneities throughout the structure. To bridge this gap, we use laser scanning confocal microscopy (LSCM) as our routine real-space imaging tool. LSCM provides further insight into our network structures as it allows $3 \mathrm{D}$ visualization of a sample. Experiments are performed with an in-house Nikon A1R MP laser scanning confocal microscope. The excitation wavelength is 402 or $457 \mathrm{~nm}$. As the IPG photoresist shows sufficient autofluorescence at both of these excitation wavelengths, further addition of a dye to the photoresist is not necessary. The sample is imaged in a glass cell filled with toluene, which ensures clear samples by matching the refractive index of the solvent $\left(n_{\text {toluene }}=1.496\right)$ and the polymer $\left(n_{\text {resist }}=1.52\right)$. Similar results are obtained using microscope immersion oil as a clearing agent. Typical results are shown in Fig. 7. The images clearly reveal the 3D network structure and each dielectric rod is visible. Images in Figs. 7(a) and 7(b) show 3D reconstructions of a $35 \times 35 \times 14 \mu \mathrm{m}^{3}$ subvolume of a sample whereas Fig. 7(c) shows a cross section of the structure, parallel to the sample surface at the height of approximately $7 \mu \mathrm{m}$. No signs of overexposure or bleaching of the autofluorescence, neither at the surface nor in the bulk of the sample, are observed. Moreover, we can use the 3D reconstruction to numerically calculate the diffraction pattern by $3 \mathrm{D}$ Fourier transformation. We note that the results of this procedure are affected by a convolution of the object with the point spread function of the microscope objective [46]. Nevertheless, as long as the structural length scales under study are large compared to the wavelength $a \gg \lambda$, we expect the additional smearing to be relatively small for our high-numerical-aperture microscope objective (Nikon NA 1.4 $100 \times$ oil-immersion objective). Indeed the results obtained by this procedure, Fig. 7(d), overall compare well to the scattering experiments and to the numerical calculations performed using the original template derived from the seed pattern as reported in Ref. [1].

\section{NUMERICAL CALCULATIONS OF THE SCATTERING FUNCTION I(q)}

\section{A. Implementation of the numerical calculations}

We model the light-scattering properties of our structures in the weak scattering limit using a discrete dipole approximation (DDA) [47]. The numerical procedure is implemented as follows: First we prepare a three-dimensional binary representation of our structures using a sufficiently densely spaced array. Following that we calculate the 3D fast Fourier transform of our dataset and then its modulus squared, which is proportional to the scattered light intensity $I(\mathbf{q})$ (or the differential scattering cross section) for a scattering vector $\mathbf{q}=\mathbf{k}-\mathbf{k}_{\mathbf{0}}$, where $\mathbf{k}$ denotes the scattered wave vector and $\mathbf{k}_{\mathbf{0}}$ is the incident
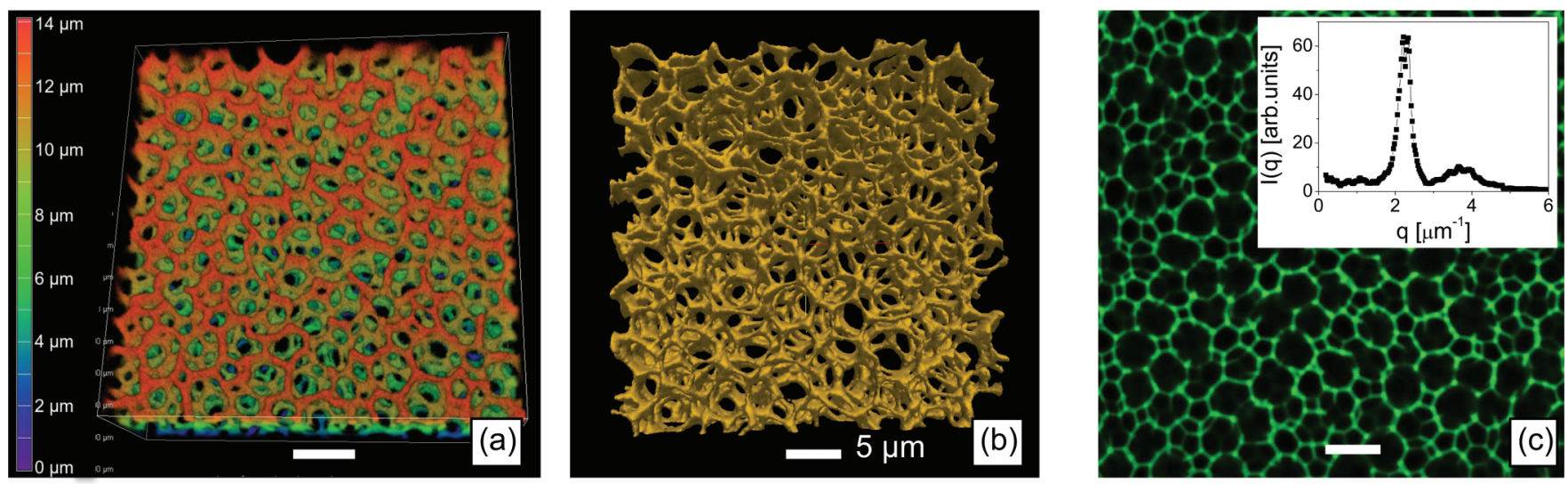

FIG. 7. (Color online) Laser scanning confocal microscopy (LSCM) images of polymer network structures with $a=3.3 \mu \mathrm{m}$. Excitation wavelength $\lambda=405$ or $457 \mathrm{~nm}$. (a) 3D rendering of a $35 \times 35 \times 14 \mu \mathrm{m}^{3}$ volume of a sample; the axial dimension is given by the color code $0 \rightarrow 14 \mu \mathrm{m}$. (b) Isosurface rendering of a different $35 \times 35 \times 14 \mu \mathrm{m}^{3}$ region in the sample. (c) A slice $\left(44 \times 44 \mu \mathrm{m}^{2}\right)$ parallel to the sample surface at the height of approx. $7 \mu \mathrm{m}$. Inset: scattering function calculated from the 3D Fourier transform of the LSCM data. All scale bars are $5 \mu \mathrm{m}$. 
wave vector. Since the structures are disordered, the scattering pattern is isotropic: A typical diffraction pattern consists of concentric rings with no distinct Bragg peaks. We thus calculate radial averages of the simulated scattering pattern, as the angular dependence carries no structural information. Note that the (numerical and experimental) diffraction pattern also shows the grainy speckle pattern characteristic of scattering from disordered materials [48]. The grid density of the DDA calculations has been chosen such that we can accurately reproduce the structure of our metamaterial while keeping the computational load at an acceptable level. Following these considerations the network structures were reproduced in silico using MATLAB [49] with a resolution of one dipole per site every $70 \mathrm{~nm}$. In order to verify the accuracy of this procedure we also carried out a small number of calculations doubling the resolution (one dipole site per $35 \mathrm{~nm}$ ). We find no significant difference and thus conclude that the accuracy of our procedure is sufficient.

\section{B. Finite-size effects}

In Ref. [1] we demonstrated that the measured and the calculated scattering functions are in good quantitative agreement. We can thus use the numerical model calculations to investigate systematically how different effects influence the scattering function of the sample. We first address the role of finite-size effects. The main limitation in size is the finite height $h \leqslant 16 \mu \mathrm{m}$ of the structures while the footprint of our structures is comparably large, with an edge length of the order of $\sim 50 \mu \mathrm{m}$. Already our first results, Ref. [1], clearly indicated that the scattering function has not completely evolved for the sample heights considered. On the other hand it is difficult to fabricate very high structures due to other competing effects such as the pyramidal distortions discussed below. Moreover, writing large volume structures is very time-consuming. We thus calculate diffraction pattern for different heights $h$, ranging from 4 to $50 \mu \mathrm{m}$ for structures with a footprint of $50 \times 50 \mu \mathrm{m}$ and $a=3.3 \mu \mathrm{m}$. Figures 8(a) and 8(b) show the radially averaged $I(q)$. The scattered intensities for each $h$ are normalized by the number of scatterers to facilitate comparison between structures of different height. We observe that the height of the primary peak, located at around $q \simeq$ $2.2 \mu \mathrm{m}^{-1}$, increases continuously with height. It can be noticed, however, that the change in peak height is most dramatic for low structures, whereas for $h \gg a$ the scattering properties asymptotically approach a bulk value. Of particular importance is the behavior at $q \rightarrow 0$ since hyperuniformity should lead to a vanishing scattering function $I(q)$ at small wave numbers. The latter appears to be a key property of the disordered photonic materials suggested by Florescu and coworkers [14]. In order to assess the low- $q$ behavior we analyze the average scattered intensity in the range between $q=0.4$ and $1.2 \mu \mathrm{m}^{-1}$ [Fig. 8(b)]. Indeed the low- $q$ scattering strongly decreases as the height of the structures is increased. Asymptotically we do not observe this contribution to tend towards zero. We think the residual scattering even for rather high structures is (at least partially) due to the finite lateral size of the structures. Due to the limited number of points in the seed pattern, extracted from Ref. [35], we have not yet been able to verify this assumption and we will address the question
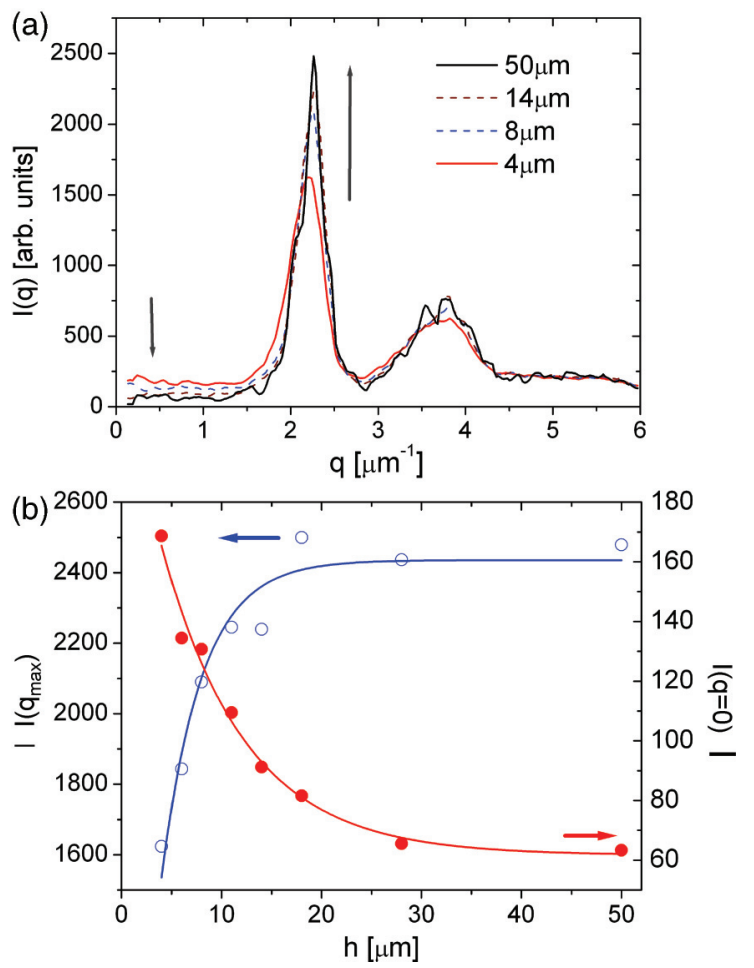

FIG. 8. (Color online) (a) Radially averaged diffraction patterns $I(q)$ derived from computer-generated 3D network structures as a function of height $h$ with $a=3.3 \mu \mathrm{m}$ (laser pen cross section $0.28 \times 0.84 \mu \mathrm{m}$ ). (b) Peak height (left axis) and low $q$ values (values averaged between 0.4 and $1.2 \mu \mathrm{m}^{-1}$, right axis) as a function of height. The footprint of the structures is $50 \times 50 \mu \mathrm{m}$.

in a future study using larger systems. The height of $I\left(q_{\max }\right)$ and $I(q \rightarrow 0)$ approaches a plateau value with an exponential decay and rise, Fig. 8(b). The characteristic lengths obtained by fitting the $h$ dependence are $h_{\text {peak }} \sim 1.2 a$ and $h_{\text {zero }} \sim 2.5 a$, respectively.

\section{Pyramidal distortions}

When writing structures higher than two or three basic units $(h \geqslant 2 a)$, we observed a pyramidal distortion, visible in scanning electron micrographs, Fig. 9(a). These distortions are most likely due to stresses that build up during polymerization and development. At the glass interface the stresses are compensated by interactions with the substrate while at larger height this leads to visible shrinkage. From electron microscopy we estimate a distortion angle of approximately $15^{\circ}$. In order to assess the influence of such unwanted deformations we calculate the diffraction patterns of both nondistorted samples (which, for the time being, we are not able to fabricate) and the distorted ones. Fortunately we find that the distortion does not significantly affect the scattering function $I(q)$ but only results in a slight shift of $I(q)$ towards larger $q$. This effect is caused by the shrinkage of the upper parts of the structure, slightly decreasing the characteristic distances inside the structure. For a $8-\mu \mathrm{m}$-high sample we estimate, from electron microscopy images, that the linear shrinkage increases from 0 close to the substrate to $7.8 \%$ at the free surface at the top, corresponding to an average shrinkage of 
(a)
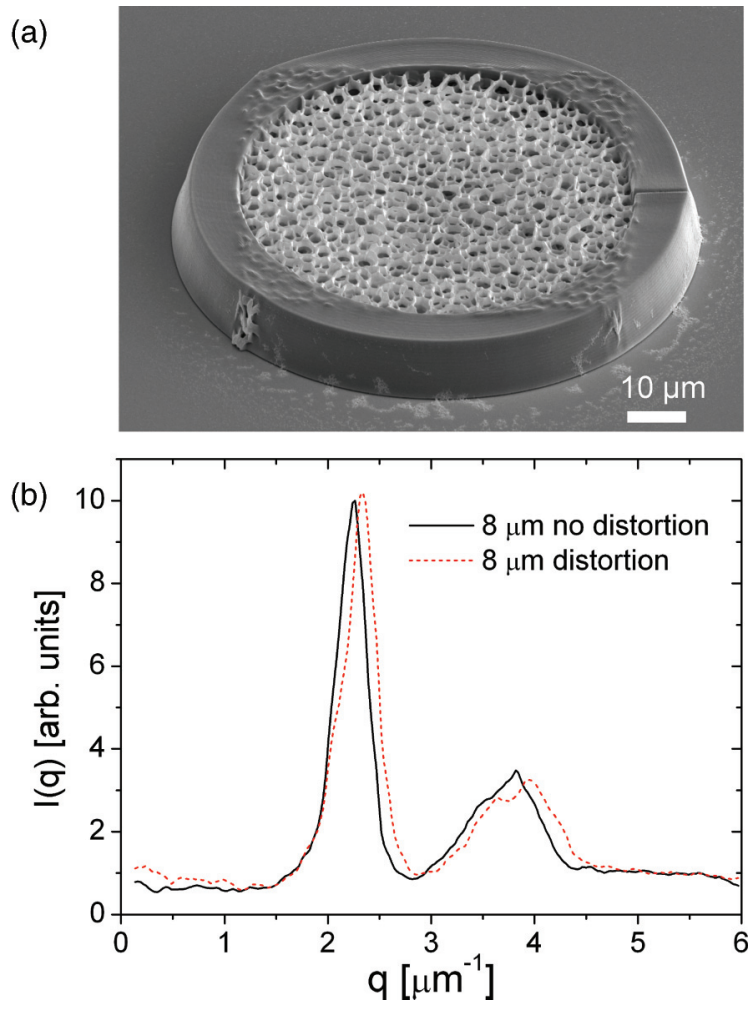

FIG. 9. (Color online) (a) Scanning electron micrographs (oblique view) of a structure of height $h=12 \mu \mathrm{m}$. (b) Scattering function $I(q)$ of a computer-generated network structure with and without a pyramidal distortion.

3.9\%. From the calculations, Fig. 9, we estimate a shift of $\Delta q \sim 0.08 \mu \mathrm{m}^{-1}$ corresponding to shrinkage of $3.5 \%$, which is in good agreement with the former value.

\section{Influence of the ellipticity of the laser pen}

The cross section and aspect ratio of dielectric rods fabricated by direct laser writing depend on the shape and size of the isointensity surface of the focused laser spot at the cross-linking threshold of the polymer photoresist [39]. The isointensity surface is given by the point spread function of the objective for the given illumination conditions. In general it is an ellipsoid, elongated in the direction of propagation of the laser beam. An important consequence of this is that the cross section of a given rod depends on its orientation in space. All rods perpendicular to the sample surface (or parallel to the beam) have a circular cross section, whereas those parallel to the surface are elliptic. We have thus examined numerically whether the light-scattering properties of a computer-generated structure are affected by this difference in rod shape within the structures. Starting from the same seed pattern we construct a network either using the elliptical pen shape with an aspect ratio of 2.9 or a spherical pen. As before we divide the calculated scattering function by the total number of dipoles. The results in Fig. 10 show that the elliptical pen shape has very little effect on the radially averaged scattering function $I(q)$. The only visible difference is the fact that the peak height is slightly increased for the case of a structure obtained with a spherical pen shape.

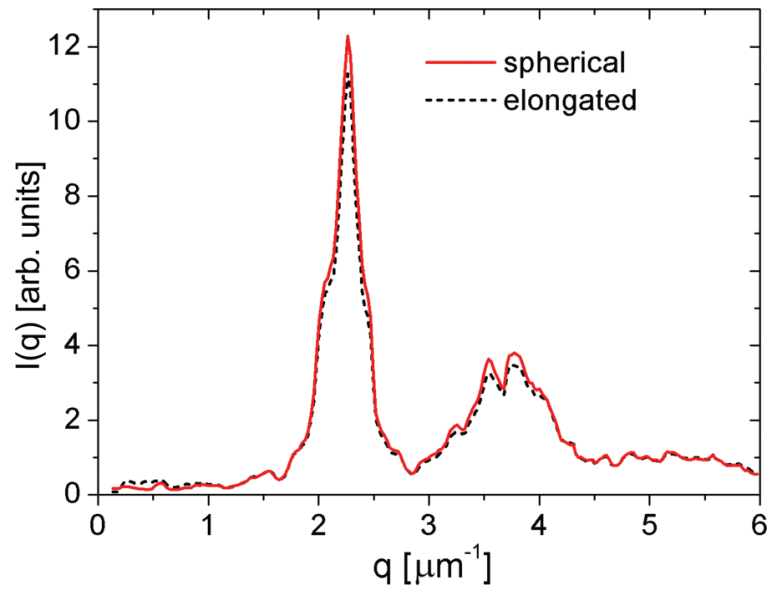

FIG. 10. (Color online) Scattering function $I(q)$ derived from computer-generated 3D network structures $(a=3.3 \mu \mathrm{m}, h=$ $50 \mu \mathrm{m})$ using either an ellipsoidal (elongated, $0.28 \mu \mathrm{m} \times 0.84 \mu \mathrm{m}$ ) or spherical (diameter $0.458 \mu \mathrm{m}$ ) laser writing pen.

\section{SUMMARY AND CONCLUSIONS}

In this paper we have presented a detailed study of the different parameters affecting the fabrication of disordered hyperuniform polymeric templates for photonic materials. Expanding on our previous work [1] we succeeded in reducing all length scales by a factor of $5 / 3$. At the current stage we are able to fabricate routinely polymeric networks starting from a seed pattern with an average point-to-point distance of $a=2 \mu \mathrm{m}$ and an average rod length of $d \simeq 0.8 \mu \mathrm{m}$ in the network after tesselation. Moreover, we have introduced and discussed several characterization tools that allow us to assess the quality of the fabricated polymer structures both in real space and in reciprocal space. A substantial part of this study has been dedicated to the analysis of the various parameters and experimental artifacts that affect the quality of the fabricated structures in practice. The numerical study shows that a minimum height $h \geqslant 3 a$ of the structure is required for the photonic properties to fully develop. For structures smaller than this finite-size effects play an important role. Our numerical studies of the scattering function $I(q)$ show that imperfections due to distortions and the ellipsoidal shape of the writing pen have only a limited impact on the scattering function. Nonetheless, further improvements in accuracy might be required when writing larger and higher structures. Also, the ellipsoidal shape might adversely affect the spectral properties when a photonic gap opens up at higher refractive index contrast. The pyramidal distortion could be reduced by prestructuring the substrate with hollow cylindrical holes using classical 2D lithography. Writing the structure inside such voids would allow connection of the outer part of the structure to a solid support that would not deform during development. A similar quality improvement could be obtained by increasing the thickness of the sturdy wall used already now to limit the deformation as shown in Figs. 4 and 9. Both strategies are currently tested in our laboratories. Reducing the aspect ratio of the laser pen on the other side always comes at the expense of reduced writing resolution. Shaping the point spread function of the laser pen with a shaded ring filter is the optimal strategy with minimal loss 
in resolution $[39,40]$. Design, alignment, and characterization are rather difficult, however, and our current implementation is still experimental (and the commercial version marketed by Nanoscribe GmbH, Germany, has been withdrawn from their product portfolio in the meantime). Another strategy consists in writing several ellipsoidal voxels side by side, thereby reducing the aspect ratio. The latter strategy is reliable and allows reducing the aspect ratio to values close to one. It does, however, also reduce the actual resolution roughly by a factor equivalent to the aspect ratio of the voxel, so in our case by about a factor 3 .

\section{ACKNOWLEDGMENTS}

We thank the MPI for polymer research (Mainz) for giving us access to their focused-ion-beam (FIB) instrument and we thank Michael Kappl for help with the experiments. We thank Frédéric Cardinaux for discussions and help preparing the manuscript. J.H. acknowledges financial support from a Sciex Swiss Research Fellowship No. 10.030. The present project has been financially supported by the National Research Fund, Luxembourg (Project No. 3093332), the Swiss National Science Foundation (Projects No. 132736 and No. 149867), and the Adolphe Merkle Foundation.
[1] J. Haberko and F. Scheffold, Opt. Express 21, 1057 (2013).

[2] J. D. Joannopoulos, Photonic Crystals: Molding the Flow of Light, 2nd ed. (Princeton University Press, Princeton, NJ, 2008); http://ab-initio.mit.edu/book/photonic-crystals-book.pdf

[3] E. Yablonovitch, Phys. Rev. Lett. 58, 2059 (1987).

[4] S. John, Phys. Rev. Lett. 58, 2486 (1987).

[5] C. Lõpez, Adv. Mater. 15, 1679 (2003).

[6] K. M. Ho, C. T. Chan, C. M. Soukoulis, R. Biswas, and M. Sigalas, Solid State Commun. 89, 413 (1994).

[7] C. M. Soukoulis and M. Wegener, Nat. Photon. 5, 523 (2011).

[8] P. Lodahl et al., Nature (London) 430, 654 (2004).

[9] L. F. Rojas-Ochoa, J. M. Mendez-Alcaraz, J. J. Saenz, P. Schurtenberger, and F. Scheffold, Phys. Rev. Lett. 93, 073903 (2004).

[10] M. Reufer, L. F. Rojas-Ochoa, P. Schurtenberger, J. J. Saenz, and F. Scheffold, Appl. Phys. Lett. 91, 171904 (2007).

[11] J. F. Galisteo-López, M. Ibisate, R. Sapienza, L. S. Froufe-Prez, A. Blanco, and C. López, Adv. Mater. 23, 30 (2011).

[12] S. Magkiriadou, J. G. Park, Y. S. Kim, and V. N. Manoharan, Opt. Mater. Express 2, 1343 (2012).

[13] P. D. Garca, R. Sapienza, and C. López, Adv. Mater. 22, 12 (2010).

[14] M. Florescu, S. Torquato, and P. J. Steinhardt, Proc. Natl. Acad. Sci. USA 106, 20658 (2009).

[15] C. J. Jin, X. D. Meng, B. Y. Cheng, Z. L. Li, and D. Z. Zhang, Phys. Rev. B 63, 195107 (2001).

[16] W. N. Man, M. Megens, P. J. Steinhardt, and P. M. Chaikin, Nature (London) 436, 993 (2005).

[17] M. Rechtsman, A. Szameit, F. Dreisow, M. Heinrich, R. Keil, S. Nolte, and M. Segev, Phys. Rev. Lett. 106, 193904 (2011).

[18] S. Torquato and F. H. Stillinger, Phys. Rev. E 68, 041113 (2003).

[19] W. N. Man, M. Florescu, K. Matsuyama, P. Yadak, G. Nahal, S. Hashemizad, E. Williamson, P. Steinhardt, S. Torquato, and P. Chaikin, Opt. Express 21, 19972 (2013).

[20] S. F. Liew, J.-K. Yang, H. Noh, C. F. Schreck, E. R. Dufresne, C. S. O’Hern, and H. Cao, Phys. Rev. A 84, 063818 (2011).

[21] P. V. Parimi, W. T. Lu, P. Vodo, J. Sokoloff, J. S. Derov, and S. Sridhar, Phys. Rev. Lett. 92, 127401 (2004).

[22] S. Yang, J. H. Page, Z. Liu, M. L. Cowan, C. T. Chan, and Ping Sheng, Phys. Rev. Lett. 88, 104301 (2002).

[23] M. Deubel, G. von Freymann, M. Wegener, S. Pereira, K. Busch, and C. M. Soukoulis, Nat. Mater. 3, 444 (2004).
[24] G. Keiser, Optical Fiber Communications, 4th ed. (McGrawHill, New York, 2011).

[25] A. Blanco, E. Chomski, S. Grabtchak et al., Nature (London) 405, 437 (2000).

[26] I. Staude, G. von Freymann, S. Essig, K. Busch, and M. Wegener, Opt. Lett. 36, 67 (2011).

[27] V. K. Varadan, X. Jiang, and V. V. Varadan, Microstereolithography and Other Fabrication Techniques for 3D MEMS (Wiley, New York, 2001), Chap. 3.

[28] M. Campbell, D. N. Sharp, M. T. Harrison, R. G. Denning, and A. J. Turberfield, Nature (London) 404, 53 (2000).

[29] S. Jeon, J. U. Park, R. Cirelli, S. Yang, C. E. Heitzman, P. V. Braun, P. J. A. Kenis, and J. A. Rogers, Proc. Natl. Acad. Sci. USA 101, 12428 (2004).

[30] S. Maruo and J. T. Fourkas, Laser Photon. Rev. 2, 100 (2008)

[31] J. Fischer and M. Wegener, Laser Photon. Rev. 7, 22 (2013).

[32] S. W. Hell and J. Wichmann, Opt. Lett. 19, 780 (1994).

[33] T. A. Klar and S. W. Hell, Opt. Lett. 24, 954 (1999).

[34] T. Böckmann, N. Stenger, M. Kadic, J. Kaschke, A. Frölich, T. Kennerknecht, C. Eberl, M. Thiel, and M. Wegener, Adv. Mater. 24, 2710 (2012).

[35] C. Song, P. Wang, and H. A. Makse, Nature 453, 629 (2008), Data taken from Hernán Makse's webpage, City College of New York (USA), http://lev.ccny.cuny.edu/ hmakse/

[36] C. E. Zachary, Y. Jiao, and S. Torquato, Phys. Rev. Lett. 106, 178001 (2011).

[37] We note that our seed patterns are hyperuniform but not stealthy (for details, see Refs. $[14,38]$ ). We currently do not have access to stealthy patterns. We plan to numerically generate such patterns in the future, which, we hope, will allow us to address the role of the degree of hyperuniformity on the spectral properties of disordered photonic materials.

[38] R. D. Batten, F. H. Stillinger, and S. Torquato, J. Appl. Phys. 104, 033504 (2008).

[39] A. Ledermann, Ph.D. thesis, Karlsruhe Institute of Technology (KIT), Germany, 2010 (unpublished), http://digbib.ubka.uni-karlsruhe.de/volltexte/1000015608

[40] M. Martinez-Corral, C. Ibanez-Lõpez, G. Saavedra, and M. Caballero, Opt. Express 11, 1740 (2003).

[41] T. Böckmann, N. Stenger, M. Kadic, J. Kaschke, A. Frölich, T. Kennerknecht, C. Eberl, M. Thiel, and M. Wegener, Adv. Mater. 24, 2710 (2012). 
[42] G. von Freyman, A. Ledermann, M. Thiel, I. Staude, S. Essig, K. Busch, and M. Wegener, Adv. Funct. Mater. 20, 1038 (2010).

[43] The effect of the shaded-ring filter has not yet been evaluated for the dip-in configuration and we have also not focused our attention on optimzing its use, which may leave some room for further improvements.

[44] M. Kerker, The Scattering of Light and Other Electromagnetic Radiation (Academic Press, New York, 1969).
[45] F. K. Tittel, D. Richter, and A. Fried, Topics Appl. Phys.: SolidState Mid-infrared Laser Sources 89, 458 (2003).

[46] J.-B. Sibarita, Adv. Biochem. Engin. Biotechnol. 95, 201 (2005).

[47] B. T. Draine and P. J. Flatau, J. Opt. Soc. Am. A 1, 1491 (1994).

[48] J. W. Goodman, J. Opt. Soc. Am. A 66, 1145 (1976).

[49] MATLAB and Statistics Toolbox Release (MathWorks, Inc., Natick, MA, 2012). 